\title{
La atención de los enfermos y discapacitados en el hogar. Retos para el sistema de salud mexicano
}

\author{
Gustavo Nigenda, PhD, (') Mariana López-Ortega, M en Pol Púb, (2) Cecilia Matarazzo, M en Antrop, ${ }^{(3)}$ \\ Clara Juárez-Ramírez, Dra en Antrop. ${ }^{(1)}$
}

Nigenda G, López-Ortega M, Matarazzo C, Juárez-Ramírez C. La atención de los enfermos y discapacitados en el hogar. Retos para el sistema de salud mexicano. Salud Publica Mex 2007;49:286-294.

\section{Resumen}

Objetivo. Identificar el patrón de dedicación de tiempo y analizar los mecanismos de atención que utilizan los miembros de familias con enfermos y discapacitados en el hogar. Material y métodos. Para cumplir con el primer objetivo se analizó la base de datos de la Encuesta Nacional de Uso de Tiempo 2002. Para cumplir con el segundo objetivo se levantó información a través de entrevistas en profundidad y grupos focales en Coahuila, Sinaloa,Zacatecas, Jalisco, Oaxaca, Yucatán y el Distrito Federal de junio a diciembre de 2004. Resultados. Se estima que I 738756 personas dedicaron tiempo a cuidar enfermos y que I 496616 se encargaron de cuidar discapacitados, la semana anterior al levantamiento de la encuesta. Existen diferencias importantes por género y nivel de escolaridad en la dedicación de horas a estas actividades. Por otra parte, los hogares tienden a reorganizarse para ofrecer atención a enfermos y discapacitados. En el hogar, son las mujeres quienes asumen la mayor responsabilidad en el cuidado. Existen diferencias importantes en la atención a enfermos y discapacitados en términos del desgaste físico y emocional que sufre el cuidador. Conclusiones. Las implicaciones de los resultados en la atención a enfermos y discapacitados son enormes para un futuro cercano. El envejecimiento de la población y el aumento de las enfermedades

\author{
Nigenda G, López-Ortega M, Matarazzo C, Juárez-Ramírez C. \\ Household care for ill and disabled persons: \\ challenges for the Mexican health care system. \\ Salud Publica Mex 2007;449:286-294.
}

\section{Abstract}

Objective.To identify the pattern of time devoted by members of Mexican households to providing care to ill and disabled family members. To analyze the mechanisms used by families to provide care to an ill or disabled member. Material and Methods. The database of the 2002 National Survey of Time Use was explored to accomplish the first objective. The second objective was accomplished by collecting primary data through in-depth interviews and focal groups in Coahuila, Sinaloa, Zacatecas, Jalisco, Oaxaca and Yucatán from June to December 2004. Results. It was estimated that I 738756 persons spent time providing care to ill persons and I 496 616 to disabled persons, over the reference period of the survey. There are important differences in the dedication of hours by gender and education level. Moreover, households tend to reorganize their structure to provide care to ill and disabled members.Women tend to have more responsibilities in the process. There are important differences in the care of ill and of the disabled in terms of the physical and emotional stress produced in the caregiver. Conclusions. The implications of results in the care of ill and disabled populations are highly relevant for the future of the Mexican health care system. Population aging and the increase of chronic diseases call for a reinforced relationship between institutional and

(I) Centro de Investigación en Sistemas de Salud, Instituto Nacional de Salud Pública. México

(2) Estudiante de doctorado en Salud Pública y Política. Escuela de Higiene y Medicina Tropical de Londres

(3) Consultora independiente

Fecha de recibido: 6 de octubre de 2006 - Fecha de aceptado: 17 de abril de 2007

Solicitud de sobretiros: Mariana López-Ortega. Instituto Nacional de Salud Pública.

Av. Universidad 655, Col. Santa María Ahuacatitlán. 62508 Cuernavaca Morelos, México.

Correo electrónico: gnigenda@insp.mx 
crónicas requieren de una mayor articulación entre el ámbito institucional de atención y el ámbito del hogar, a fin de que ambos puedan complementar sus capacidades.

Palabras clave: Cuidadores, cuidados domiciliarios de salud, género y salud; México household care so as to complement capacities, a situation already taking place in other countries.

Key words: Home care; gender perspective; socio-economic analysis; Mexico
$\mathbf{E}$ n la actualidad, y a partir del aumento en la demanda de atención de las enfermedades crónico-degenerativas, los cambios demográficos y epidemiológicos de las poblaciones en los países industrializados han generado una gran presión sobre los servicios de salud. Estos últimos han respondido con un conjunto de estrategias, generalmente sustentadas en mecanismos financieros cuidadosamente diseñados para desarrollar respuestas adecuadas a estas necesidades. ${ }^{1}$

En un primer momento, la estrategia consistió en reducir los costos hospitalarios dando de alta lo más tempranamente posible a los pacientes. ${ }^{2}$ Sin embargo, la experiencia mostró que delegar la atención de enfermos y convalecientes en los miembros del hogar no bastaba para retenerlos en ese ámbito y evitar un reingreso institucional, lo cual implicaba una transferencia de los riesgos económicos y de salud a las familias. Un componente clave de muchas de esas estrategias ha sido el fortalecimiento de la interfase entre la institución hospitalaria, el ámbito del hogar y la comunidad. Las estrategias en los países desarrollados son muy diversas y van desde el montaje de mecanismos de aseguramiento y el pago a miembros del hogar para atender a un familiar enfermo, hasta la contratación de cuidadores profesionales que atiendan al enfermo en su propio domicilio. ${ }^{3}$

La población de naciones de ingresos medios como México ha entrado en una fase acelerada de envejecimiento que, en pocos años, generará los efectos que los países industrializados viven de lleno hoy en día. El problema de estas naciones es que la presión de una población envejecida y con altas prevalencias de enfermedades crónicas se suma a los rezagos de atención a la salud-enfermedad, comunes en muchas de ellas . ${ }^{4}$ Este problema está marcado por importantes inequidades en la distribución de las tareas de cuidado que requieren los enfermos o convalecientes, una vez que están en casa. Una de las más importantes es la de género, que prevalece en gran cantidad de hogares y se expresa básicamente a través de la dedicación de un mayor número de horas por parte de las mujeres en la casa al cuidado de enfermos y discapacitados de todas las edades. ${ }^{5}$ Es común que sean las mujeres quienes se encarguen de este tipo de actividades, a pesar de que en los últimos 25 años se han dado notables cambios en la estructura y composición de los hogares mexicanos. Lo anterior es resultado, entre otras cosas, de la mayor participación femenina en el mercado laboral (formal e informal) y de un incremento en su acceso a oportunidades de educación, todo lo cual ha generado cambios en la composición de la estructura familiar tradicional ${ }^{6} \mathrm{y}$ en la distribución de las tareas y responsabilidades entre los miembros del hogar. ${ }^{7}$ Por otra parte, la reducción de las tasas de fecundidad, el retraso en la primera unión y el aumento de hogares sin hijos o monoparentales, son cambios que afectarán en el mediano y largo plazo la disponibilidad de personas en el hogar que dediquen tiempo al cuidado de enfermos y discapacitados. Los cambios en el perfil demográfico, epidemiológico y social del país, así como la reducción de las tasas de fecundidad y el incremento en la población mexicana de personas mayores de 60 años ${ }^{*, 8}$ implican que sus necesidades en términos de pensiones, servicios sociales y servicios de salud deberán crecer a un ritmo similar si lo que se busca es cubrirlas cabalmente. No obstante, el ambiente económico que prevalece en México hoy en día augura una situación complicada en el futuro, ya que de los individuos los mayores de 60 años sólo $29 \%$ tienen un empleo, apenas $2.7 \%$ de los hombres y $1.6 \%$ de las mujeres cuentan con una pensión y se estima que $20 \%$ viven en condiciones de pobreza. ${ }^{9}$

La esperanza de vida en México ha aumentado en las últimas décadas de tal manera que es de 73 años para los hombres y 77 años para las mujeres. La prolongación del periodo de vida representa un reto para los sistemas de salud y protección social, ya que deben contender con enfermedades de más larga duración cuyos costos por individuo son mucho más altos que aquellos de las enfermedades infecciosas. ${ }^{10}$ A partir de la Encuesta Nacional sobre Uso del Tiempo 2002 (ENUT

\footnotetext{
* En el año 2005, este grupo de población sumó alrededor de 8.2 millones de personas y se estima que para el 2030 serán alrededor de 22.2 millones. Proporcionalmente este grupo pasará de representar el $8 \%$ de la población a más del 25\% (Nigenda G, Matarazzo C, López-Ortega M. Los cuidados a la salud en el hogar: tendencias e inequidades. Análisis desde una perspectiva de género. FunsaludSSA. México DF, 2005).
} 
2002), Pedrero ${ }^{11}$ destaca la participación de hombres y mujeres en distintas actividades domésticas y el tiempo promedio dedicado a cada una de éstas. Así por ejemplo, señala que la limpieza del hogar es la actividad a la que más mujeres (93.1\%) dedican tiempo, a diferencia de los hombres entre quienes sólo la mitad $(48.7 \%)$ realiza estas tareas. Además, las mujeres dedican en promedio al día 14 horas y 27 minutos a la limpieza del hogar, en tanto que los hombres le destinan únicamente 4 horas y 24 minutos. Otra labor relevante es la preparación de alimentos, donde las mujeres invierten 2.5 veces más tiempo que los hombres.

En conjunto, cocinar, limpiar y llevar a cabo el cuidado de la ropa ocupan más de $63 \%$ del tiempo que las mujeres dedican al trabajo doméstico, tanto para aquellas que realizan trabajo extra doméstico como para las que no lo hacen. Al sumarse el tiempo destinado al cuidado de los niños, las actividades de las mujeres llegan a representar casi $80 \%$ del uso total de su tiempo. Pedrero afirma que, con excepción de las reparaciones y actividades de mantenimiento, donde los hombres superan a las mujeres (70 y $30 \%$, respectivamente), no hay ninguna otra actividad a la que los hombres le dediquen mayor tiempo. Adicionalmente a las horas destinadas a las tareas domésticas y extra domésticas, se agregan aquellas dedicadas a las diversas actividades que implica el cuidado a la salud de los miembros del hogar que lo necesitan. Lo anterior tiene implicaciones relevantes para la salud física y emocional de aquellas mujeres que, a su rol doméstico, agregan el de ser cuidadoras de familiares enfermos. Así entonces, no es raro encontrar que las personas que cuidan de familiares enfermos vean reducidas sus posibilidades de seguir estudiando o trabajando, como consecuencia del costo de oportunidad que tienen que pagar por asumir esas tareas.

A pesar de que son evidentes los problemas que, en el futuro puede implicar el crecimiento acelerado de la población de adultos mayores, en México no se han diseñado estrategias para enfrentarlos desde la perspectiva social, cultural, e institucional. Los esfuerzos de diversas instituciones como el IMSS $^{12}$ o el ISSSTE ${ }^{13}$ se ubican apenas en la etapa de reconocimiento del fenómeno y de su aceptación. El presente trabajo es producto de una investigación cuyo objetivo fue documentar los tiempos dedicados en los hogares para realizar actividades relacionadas con los cuidados a la salud y los mecanismos que los miembros de los hogares desarrollan para ofrecer esa atención. Como resultado de esa indagación se busca argumentar a favor de la necesidad de fortalecer el vínculo entre la institución, la comunidad y el hogar, a manera de mecanismo que provea atención adecuada a grupos de población envejecidos, buscando que la atención sea compartida de acuerdo con las capacidades y que, además, ayude a reducir inequidades pre-existentes en la atención. *

\section{Material y métodos}

En 2004 se llevó a cabo un estudio con el objeto de documentar la atención a enfermos crónicos en el ámbito del hogar. ${ }^{\ddagger}$ El diseño metodológico contempló una etapa cuantitativa y otra cualitativa. En la primera se llevó a cabo un análisis de la base de datos de la ENUT 2002,14 para estimar tiempos de dedicación a distintas actividades en una muestra con representatividad nacional, tanto urbana como rural. ${ }^{\S}$ Para el levantamiento de la ENUT 2002 se obtuvo una submuestra de la Encuesta Nacional de Ingreso y Gasto de los Hogares 2002 (ENIGH 2002) de 6288 viviendas seleccionadas, a partir de la cual se identificaron 6,398 hogares, de los cuales se encuestaron $5450 .{ }^{15}$ La información se recolectó mediante un cuestionario con actividades predefinidas en preguntas cerradas, aplicado de manera directa a cada uno de los miembros del hogar de 12 años de edad y más. Del cuestionario de la ENUT se identificaron aquellas preguntas relacionadas con el número de horas dedicadas tanto por los hombres como por las mujeres a realizar diferentes actividades de cuidado a enfermos y discapacitados dentro del hogar. Se identificaron las principales características sociodemográficas de las personas que realizaban los cuidados, entre las que destacan el nivel de educación, la condición laboral y el nivel de ingresos.

De acuerdo con la ENUT 2002, el cuidado a enfermos, que en adelante se denominará como cuidados a la salud en el hogar, se define como aquella atención que se da a los miembros del hogar que presentan alguna enfermedad, ya sea en la vivienda o en una unidad de salud. Adicionalmente, se define el cuidado de personas con limitaciones físicas o mentales como las actividades en apoyo y cuidado de miembros del hogar con limitaciones físicas o mentales, tales como ayudarles a comer, bañarse y vestirse; aplicarles alguna terapia; y, por último, llevarlos o acompañarlos al servicio médico o a realizar algún trámite. En adelante

\footnotetext{
* Mismas que se incrementarán en los próximos años por los cambios recientes en la estructura demográfica y epidemiológica del país.

* La Fundación Mexicana para la Salud llevó a cabo esta investigación con fondos de la Secretaría de la Salud.

$\S$ La ENUT se levanta a solicitud de las instituciones interesadas y, hasta la fecha, no es periódica. Permite conocer la participación y el tiempo que los hombres y mujeres de 12 años y más le dedican a cada una de las actividades domésticas y extradomésticas.
} 
se hará referencia a esta actividad como el cuidado a discapacitados.

En este trabajo se muestran los patrones generales de dedicación de tiempo, a través de un primer análisis descriptivo de las variables de la ENUT 2002* complementado con información primaria derivada de trabajo de campo en Coahuila, Sinaloa, Zacatecas, Jalisco, Oaxaca, Yucatán y el Distrito Federal. La información cuantitativa se presenta como frecuencias y tabulados de las variables seleccionadas tomando como variables para el análisis el sexo, nivel educativo, nivel de ingresos y condición laboral. ${ }^{\ddagger}$ Asimismo, se realizaron pruebas estadísticas para comprobar independencia de las variables y diferencia de proporción de poblaciones. El análisis se llevó a cabo con el paquete estadístico STATA ${ }^{\S}$ y con un nivel de significancia estadística de 95\%.

La fase cualitativa se realizó en las entidades antes mencionadas. El objetivo fue indagar de primera mano y con mayor detalle las experiencias de las familias y de las instituciones en relación con el cuidado de enfermos

\footnotetext{
* Durante el periodo de aplicación de la encuesta, los entrevistadores acuden a los hogares seleccionados para entrevistar, de nueva cuenta, a las familias y obtener información sobre las tareas que llevaban a cabo los miembros del hogar en ese ámbito, así como las horas que les dedican, siendo la última semana el tiempo de referencia. Sólo los miembros de los hogares mayores de 12 años responden el cuestionario.

₹ La variable de nivel educativo se codificó nuevamente para quedar con cinco niveles: sin educación formal, educación primaria, educación secundaria, bachillerato/preparatoria, y licenciatura/ posgrado.

$\S$ Stata Corp. STATA, Statistical Software 8.2. College Station. Texas, EUA. 2003.
}

crónicos. Los estados visitados se seleccionaron a partir de la identificación de características sociodemográficas como: a) el nivel de envejecimiento de la población, b) perfil epidemiológico, c) grado de marginación y d) ubicación geográfica. Se utilizaron técnicas de investigación como los grupos focales y las entrevistas en profundidad de orientación biográfica. Ambas se llevaron a cabo con usuarios de los servicios de salud de la Secretaría de Salud Estatal. Se formaron 13 grupos focales, con un mínimo de seis y un máximo de 11 participantes. Asimismo se recuperaron testimonios de 25 funcionarios responsables de programas de salud dirigidos a población envejecida (SSA, ISSSTE e IMSS).

Para las dos técnicas empleadas se diseñaron guías de entrevista semiestructurada. Tanto los grupos focales como las entrevistas fueron grabadas (previo consentimiento informado), transcritas en el programa Word y procesadas con el software Atlas Ti. ${ }^{*}$ Las mujeres entrevistadas de manera individual fueron seleccionadas por ser los casos más representativos de los grupos focales, además de que aceptaron dedicar tiempo para profundizar en el tema de estudio. Los temas indagados se agruparon en dos grandes categorías: experiencia personal del cuidado al enfermo en el hogar (tiempo dedicado al cuidado, habilidades adquiridas, capacitación formal, percepción del problema, impacto en la vida personal) y expectativas sobre el apoyo institucional (gasto en salud, capacitación, calidad del servicio). Con los funcionarios se indagó

* Muhr T. Atlas Ti. Scientific software development. Berlín, Alemania 1998.

Cuadro I

Distribución de horas de atención en el hogar a enfermos, por SeXo y niVel de escolaridad. MéXiCo, 2002

\begin{tabular}{|c|c|c|c|c|c|c|}
\hline \multirow[b]{2}{*}{ Nivel educativo } & \multicolumn{3}{|c|}{ Mujeres } & \multicolumn{3}{|c|}{ Hombres } \\
\hline & Total & $\%$ del nivel & Promedio de hrs/semana & Total & $\%$ del nivel & Promedio de hrs/semana \\
\hline Sin educación formal & 111378 & 78.8 & 11.67 & 29922 & 21.2 & 16.07 \\
\hline Educación primaria & 389517 & 70.5 & 9.02 & 163262 & 29.5 & 9.10 \\
\hline Educación secundaria & 457574 & 80.3 & 4.54 & 112880 & 19.7 & 5.60 \\
\hline Bachillerato/Preparatoria & 123511 & 60.9 & 4.90 & 79511 & 39.1 & 4.59 \\
\hline Licenciatura/ Posgrado & 120790 & 45 & 7.15 & |48 05 | & 55 & 8.53 \\
\hline Total & I 202770 & 69.2 & & 533626 & 30.8 & \\
\hline \multicolumn{7}{|c|}{ Fuente: ENUT 2002 y ENIGH 2002} \\
\hline
\end{tabular}


Cuadro II

Distribución DE hORAS de ATENCIÓN EN EL HOGAR A ENFERMOS, POR SEXO Y QUINTIL DE INGRESO EN LA FAMILIA. MÉXICO, 2002

\begin{tabular}{|c|c|c|c|c|c|c|}
\hline \multirow[b]{2}{*}{ Ingresos } & \multicolumn{3}{|c|}{ Mujeres } & \multicolumn{3}{|c|}{ Hombres } \\
\hline & Total & \% del quintil & promedio horas/ semana & Total & $\%$ del quintil & promedio horas/ semana \\
\hline I & 166391 & 70 & 8.4 & 71056 & 30 & 4.3 \\
\hline 2 & 313679 & 73.5 & 9.9 & 112565 & 26.5 & 9.9 \\
\hline 3 & 403512 & 74.9 & 5.8 & 135204 & 25.1 & 10.1 \\
\hline 4 & 231776 & 67.1 & 4.4 & 113510 & 32.9 & 7.6 \\
\hline 5 & 89772 & 46.9 & 5.6 & 101291 & 53.1 & 5.7 \\
\hline Total & $|205| 30$ & 69.3 & & 533626 & 30.7 & \\
\hline
\end{tabular}

sobre la política institucional de atención a población envejecida, percepción sobre la atención en el hogar de enfermos crónicos y sobre requerimientos futuros estatales para cubrir la demanda de atención.

\section{Resultados}

\section{La encuesta}

De acuerdo con la ENUT 2002, un total de 1738756 personas dedicaron 12 millones de horas a cuidar enfermos en sus hogares, con un promedio de 6.09 horas por persona. Existen diferencias notables en relación con la distribución de horas de acuerdo con el género, el nivel educativo y la estructura de ingresos. Un aspecto inicial que cabe señalar es que dos terceras partes de las horas dedicadas a la atención a enfermos corresponden al cuidado que brindan las mujeres. En el cuadro I se observa que, en lo tocante a la distribución de horas de cuidados a enfermos entre hombres y mujeres por nivel de escolaridad la mayor proporción de cuidadores se concentra entre las mujeres en todas las categorías de escolaridad, con excepción de la correspondiente a licenciatura/posgrado, donde los hombres representan $55 \%$ de los cuidadores. Otro aspecto que cabe destacar es que en los grupos con menor escolaridad, independientemente del género, se observan los promedios de horas de dedicación semanal más altos. Este promedio de horas se reduce en la medida en que se eleva el nivel de escolaridad, excepto en aquel de licenciatura/ posgrado.

En el cuadro II se muestra la distribución de dedicación de horas de atención a enfermos, entre hombres y mujeres, de acuerdo con el quintil de ingresos de la población. Esta distribución es similar a la anterior, ya que en todos los niveles de ingreso las mujeres cuidadoras representan la mayoría ubicándose entre 67 y 75\% respecto de los hombres. Sin embargo, los promedios de horas semanales dedicadas en cada grupo varían respecto a la distribución anterior. En este caso, tienden a tener promedios más altos que las mujeres, excepto en el quintil más bajo.

En el caso de los brindados a personas discapacitadas, el total de personas que declararon su participación en esas actividades fue de 1496616 y el número de horas de 12231714 , con un promedio de 8.17 horas por persona, cifra $34 \%$ más alta que el promedio de horas dedicadas por los cuidadores de enfermos. En el cuadro III se muestra la distribución de horas de cuidados a discapacitados, entre hombres y mujeres por nivel de escolaridad; se puede observar que, en todos los niveles de escolaridad, es mayor el porcentaje de mujeres que dedican tiempo a la atención de discapacitados que el de hombres. En todos los casos, la proporción de mujeres es cercana a $80 \%$, en tanto que en educación primaria y licenciatura/posgrado, la cifra es similar en ambos grupos. Los promedios de horas de dedicación a la semana son más altos para las mujeres en comparación con los hombres, en particular en los grupos con bajo nivel educativo, donde la cifra rebasa las 10 horas diarias en el caso de las mujeres.

Al observar la distribución por quintil de ingreso en el cuadro IV, la distribución resulta ligeramente distinta a la del cuadro III: en primer lugar, en todas las categorías de ingresos el porcentaje de mujeres que atienden a discapacitados es significativamente más alto que el de hombres, destacando las diferencias en los quintiles 1,3 y 5 . Si se considera el promedio de horas dedicadas en promedio 
Cuadro III

Distribución de horas de ATENCIÓn en El hogar a discapacitados, POR SEXo Y NIVEl de eSCOLARIDAD. MéXICO, 2002

\begin{tabular}{|c|c|c|c|c|c|c|}
\hline \multirow[b]{2}{*}{ Nivel educativo } & \multicolumn{3}{|c|}{ Mujeres } & \multicolumn{3}{|c|}{ Hombres } \\
\hline & Total & $\%$ del nivel & Promedio horas/ semana & Total & $\%$ del nivel & Promedio horas/ semana \\
\hline Sin educación formal & $20 \mid 231$ & 84.1 & 11.91 & 37985 & 15.8 & 7.5 \\
\hline Educación primaria & 402920 & 53.3 & 10.71 & 352581 & 46.6 & 4.62 \\
\hline Educación secundaria & 222020 & 79.3 & 8.32 & 57943 & 20.6 & 4.26 \\
\hline Bachillerato/Preparatoria & 54878 & 76.8 & 4.23 & 16500 & 23.1 & 1.85 \\
\hline Licenciatura/ Posgrado & 86001 & 57.1 & 10.27 & 64557 & 42.8 & 5.67 \\
\hline Total & 967050 & 64.6 & & 529566 & 35.4 & \\
\hline
\end{tabular}

Fuente: ENUT 2002 y ENIGH 2002

Cuadro IV

Distribución DE hORAS de ATENCIÓN EN EL HOGAR A DISCAPACITADOS, POR SEXO Y QUINTIL DE INGRESO DE LA FAMILIA. MÉXICO, 2002

\begin{tabular}{|c|c|c|c|c|c|c|}
\hline \multirow[b]{2}{*}{ Ingresos } & \multicolumn{3}{|c|}{ Mujeres } & \multicolumn{3}{|c|}{ Hombres } \\
\hline & Total & \% del quintil & Promedio horas/ semana & Total & $\%$ del quintil & Promedio horas/ semana \\
\hline I & 189474 & 78.2 & 11.13 & 52817 & 21.7 & 5.52 \\
\hline 2 & 397542 & 58 & 10.77 & 287536 & 42 & 5.04 \\
\hline 3 & 217567 & 68 & 7.19 & 101949 & 32 & 3.52 \\
\hline 4 & 107537 & 60 & 14.13 & 71554 & 40 & 4.97 \\
\hline 5 & 54930 & 77.7 & 3.69 & 15710 & 22.2 & 6.52 \\
\hline Total & 967050 & 64.6 & & 529566 & 45.4 & \\
\hline
\end{tabular}

Fuente: ENUT 2002 y ENIGH 2002

por persona, se observa que en todos los quintiles, excepto en el más rico, el promedio calculado para mujeres es dos y hasta tres veces mayor que para los hombres.

Las tendencias mostradas en los cuadros anteriores muestran que la distribución de las horas dedicadas a enfermos y discapacitados por parte de los miembros del hogar tiene una clara composición por género. Asimismo, se observa que los individuos pertenecientes a los niveles educativos y de ingresos más bajos tienden a dedicar un mayor número de horas en promedio que aquellos que se encuentran en los niveles altos.

Como parte del análisis se realizaron pruebas t de diferencia de medias o proporciones para conocer las diferencias estadísticas entre los grupos que declararon realizar actividades de cuidados. El primer análisis in- cluyó la diferencia entre aquellos que trabajaban y los que no trabajaban al momento de prestar los cuidados. No se encontraron diferencias significativas ni para el caso de aquellos que realizaron cuidados a la salud, ni en aquellos que realizaron cuidados a discapacitados, por condición de trabajo. Adicionalmente, se buscaron diferencias por sexo entre el grupo de personas que declararon realizar cuidados. Las pruebas de diferencia de medias indican que existen diferencias significativas por sexo para el grupo que realizó cuidados a discapacitados $(p<.001)$, pero que, por el contrario, no hay ninguna diferencia significativa en el grupo que realizó cuidados a la salud. Al analizar si existen diferencias significativas entre el grupo de personas que realizaron cuidados por quintil de ingreso y nivel educativo, 
se encontró que no hay diferencias significativas por quintil de ingreso entre aquellos que declararon brindar cuidados a discapacitados; en el caso de aquellos que prestaron cuidados a la salud, sólo existen diferencias significativas entre el tercer y cuarto quintil de ingresos $(p<.001)$. Finalmente, al analizar diferencias entre el grupo de personas que realizaron cuidados por nivel de escolaridad, no se encontraron diferencias significativas ni en el caso de aquellos que cuidaron discapacitados, ni en los que brindaron cuidados a la salud.

En estos patrones de distribución de dedicación de horas subyacen fenómenos complejos de la atención médica en el hogar que obedecen a una serie de aspectos culturales y sociales relacionados con la distribución de responsabilidades y tareas entre los miembros del hogar. Si bien en México es socialmente aceptado que sean las mujeres quienes tengan una carga mayor en todas las actividades del hogar, el cuidado de enfermos crónicos y discapacitados podría tener ya un efecto sobre las formas típicas en las que se establecen las relaciones familiares, readecuando funciones, tiempos y cargas laborales de acuerdo con las nuevas necesidades que se crean a partir de la atención a un enfermo crónico en el hogar.

\section{La indagación cualitativa}

Desde esta perspectiva fue posible recoger testimonios relativos a la organización familiar para cuidar enfermos crónicos. Del análisis de la experiencia personal del cuidador, destaca la noción de los entrevistados respecto a las diferencias en los cuidados dependiendo del tipo de enfermo ya que, según su experiencia, no es lo mismo atender en casa a un enfermo agudo que a un crónico o a un discapacitado. Los informantes señalaron que a diferencia del cuidado de enfermos agudos, el de los crónicos y discapacitados implica una dedicación casi permanente durante el tiempo en que el familiar permanece en esa condición, y eso obliga a la familia a reestructurar completamente sus actividades cotidianas para atender a un enfermo que requiere vigilancia permanente. De allí que los entrevistados consideren que el hecho de tener en casa a un enfermo discapacitado constituye una de las situaciones de mayor tensión y sufrimiento para la familia. Al respecto, el patrón típico que se encontró en el estudio es que una mujer del hogar asume la responsabilidad principal del cuidado y es, además, quien asigna a otros miembros del hogar las tareas secundarias que se requieren para el cuidado del enfermo. En este sentido, al trabajo que la mujer desempeña dentro del ámbito doméstico se adiciona la responsabilidad de organizar las actividades alrededor de la atención que requiere la persona que recibe el cuidado. Lo anterior representa una carga física y emocional importante, sobre todo en aquellas mujeres que no disponen de una red amplia de apoyo social que pueda hacerse cargo del cuidado del enfermo mientras ellas están ausentes. La complicación en las actividades diarias que trae consigo el cuidado de enfermos en el hogar puede generar maltrato emocional o negligencia en la vigilancia que requieren los pacientes; sin embargo, este es un tema que difícilmente fue reconocido en los grupos focales y que sólo se mencionó de manera indirecta cuando se exploró con mayor detalle durante las entrevistas.

Se encontró que tanto en los hogares nucleares como en los extensos, es común que el papel de cuidadora de enfermos recaiga en la madre de familia. El resto de los miembros del hogar ejecutan tareas subsidiarias ya sea en el cuidado directo del enfermo o apoyando en tareas complementarias al integrante de la familia que se ocupa de la atención directa. Si bien las mujeres asumen las tareas del cuidado más directo como son la alimentación, el aseo y la aplicación de terapias, los hombres también dedican porciones importantes de su tiempo, aunque en general sólo realizan tareas complementarias. Al explorar la experiencia de los varones como cuidadores, la mayoría de los participantes de los grupos focales compartieron la noción de que los hombres no están capacitados para realizar cuidados ya que son emocionalmente débiles ante la enfermedad y no demuestran compromiso personal para llevar a cabo el cuidado directo, que es sumamente demandante y desgastador.

Los efectos que el cuidado de un enfermo crónico pueden tener sobre un núcleo familiar y sus integrantes suele ser devastador. Las personas que cuidan, particularmente las mujeres, incurren en costos de oportunidad al tener que dejar de estudiar, trabajar o utilizar tiempos para su esparcimiento debido a la extensión de tareas que implica el cuidado del paciente. Incluso, sus relaciones familiares con sus hijos y/o cónyuges se ven seriamente afectadas. Además, el desgaste impacta negativamente en la salud tanto física como emocional de los cuidadores*. Otro aspecto que vale la pena destacar es que el cuidado es asumido como parte de una responsabilidad moral y

\footnotetext{
* El siguiente testimonio ilustra la interpretación de los autores del artículo con respecto a esas situaciones: "Cuidé a mi esposo diabético durante dos años. Fuimos a médicos naturistas porque no tenía seguro (social) y la dieta a él nunca le gustó. Su enfermedad fue avanzando hasta que poco a poco empezó a inflamarse de los pies y la vista la fue perdiendo, hasta que ya no pudo caminar, luego llegó a la diálisis, pero en silla de ruedas... yo era la mujer, la esposa y tenía que aventarme el paquetito porque mis hijos estaban chicos, estudiando y trabajando. La que tenía que hacerlo era yo forzosamente, es una etapa, la más difícil de mi vida que no le deseo a nadie. Le cuidaba la dieta (pero además) había que bañarlo, llevarlo al baño, cambiarlo..." (Oaxaca 2).
} 
emocional para con los familiares. Desprenderse de la responsabilidad de cuidar a un enfermo crónico no es una opción y es considerado moralmente inaceptable. En este sentido, lo más común es que sean los padres quienes requieran de esta atención, lo cual representa una carga simbólica importante para los hijos, quienes consideran que el cuidado es parte de sus deberes filiales pese al riesgo de perder una parte importante de su desarrollo personal.

\section{Sobre la construcción de un vínculo institución-hogar para la atención de enfermos y discapacitados}

En este aspecto, los entrevistados manifestaron la necesidad de contar con orientación institucional para capacitarse con relación al cuidado de un enfermo, ya que la mayoría señaló haber desarrollado esta habilidad mediante observación directa. Pocas personas afirmaron haber recibido una instrucción especial para llevar a cabo esa tarea. Otro tema que surgió fue la necesidad de agilizar los trámites para la consulta externa y para la hospitalización, ya que hubo consenso acerca de las dificultades que se presentan a este respecto y del maltrato que implican, tanto para la familia como para el enfermo, largos tiempos de espera. Un hallazgo relevante fue la descripción de la trayectoria que un paciente crónico no derechohabiente debe seguir para conseguir atención médica. Al respecto se encontró que los diabéticos, en particular, recurren a la medicina alternativa por su bajo costo y que ese recurso constituye, tanto para la cuidadora como para la familia, una red alternativa de atención que se utiliza para aminorar la carga impuesta y para disponer de tiempo para realizar otra actividad emergente.

\section{Conclusiones}

En los últimos años México ha desarrollado estrategias innovadoras para aumentar el gasto público en salud; dicha inversión ha sido enfocada, en gran medida, a reducir los rezagos históricos de la atención a poblaciones pobres. ${ }^{16}$ Sin embargo, si se considera la velocidad a la que envejece la población mexicana, en los próximos años será necesario dedicar más recursos para la atención de la salud de este grupo poblacional tanto en las instituciones como en la atención que se brinda en los hogares. ${ }^{*}$

\footnotetext{
* En las instituciones de seguridad social se destina alrededor de 25\% de los recursos asignados a la atención directa para la población mayor de 65 años (que representa 10\%) en estas instituciones. De acuerdo con la tendencia al envejecimiento, para 2025 el gasto dedicado a los mayores de 65 años representará $50 \%$ del total en instituciones de seguridad social.
}

Las autoridades nacionales reconocen el impacto que tendrá el proceso del envejecimiento sobre la demanda de servicios para los próximos años, pero hasta la fecha no ha sido posible articular una política que reduzca los rezagos y, al mismo tiempo, genere una respuesta prospectiva al tema de la atención a los adultos mayores y a los enfermos crónicos.

De acuerdo con los datos mostrados previamente, al interior de los hogares son las mujeres quienes asumen la responsabilidad principal en la atención de adultos mayores y enfermos crónicos, tanto en la proporción en que participan con respecto a los hombres, como en el número promedio de horas dedicadas. Asimismo, a través del análisis de la encuesta se observa que la dedicación de horas varía de manera importante entre el cuidado a enfermos y el cuidado a discapacitados. Este último tipo de cuidado requiere de mayor atención por parte de un miembro de la familia. Son las mujeres, y no los hombres, quienes particularmente tienden a aumentar su promedio de dedicación en la atención a un discapacitado en comparación con la atención a un enfermo. A diferencia del cuidado a enfermos donde, en los niveles educativos y de ingresos altos, aumenta considerablemente la participación de los hombres, en el cuidado a discapacitados el papel de la mujer sigue siendo muy relevante, sin importar el nivel educativo o de ingresos.

Los datos etnográficos permiten destacar que los cuidadores desempeñan un papel fundamental para mantener la salud de los enfermos o discapacitados en el hogar pero que, al mismo tiempo, esta dedicación implica riesgos importantes para el cuidador en términos de sus oportunidades para estudiar, trabajar o llevar una vida familiar en un ambiente de distensión. En este fenómeno, las instituciones deben ocupar un lugar mucho más relevante que el que hasta ahora han tenido. En gran medida, la capacitación de un cuidador en el hogar y el apoyo directo del personal de salud podrían derivar del vínculo con las instituciones, lo cual redundaría en una mejor atención. Por otro lado, los recursos sociales y económicos disponibles en las distintas instituciones podrían utilizarse de mejor manera si se incorporara el nivel comunitario, por ejemplo, identificando otras agrupaciones que colaboran en el cuidado del enfermo y que proporcionan apoyo emocional a la familia, identificando familias con enfermos crónicos o discapacitados y retomando la experiencia de los cuidadores para diseñar una estrategia de "capacitación" y "especialización" en el cuidado del enfermo. Es posible que acciones como estas permitan una distribución más equitativa de las tareas relacionadas con los cuidados, además de mejorar el estado de salud del enfermo. ${ }^{12,13}$ 
A partir de los resultados de esta investigación, se considera que la creación y el fortalecimiento de una interfase entre las instituciones y la participación del nivel comunitario-hogar, constituye una de las estrategias que deben desarrollarse para responder a un problema que, en los próximos años, ejercerá una presión significativa en las finanzas institucionales y en su capacidad para brindar servicios. Empero, en la actualidad no parecen existir las condiciones necesarias para el desarrollo en el corto plazo de esta interfase, ya que estos dos ámbitos de atención a la salud carecen, hasta la fecha, de vínculos formales, a diferencia de lo que sucede en otras naciones. ${ }^{18}$

En México, los intentos por construir un vínculo funcional con las comunidades y las familias de los enfermos y discapacitados son sumamente incipientes; sin embargo, es necesario consolidarlos mediante un proceso de planeación, dado que la demanda crecerá exponencialmente en los próximos años. Las familias brindan cuidados al interior del hogar que no sólo impactan en las horas de atención prestada, sino también, y en el largo plazo, en el costo de oportunidad y en la propia salud de los cuidadores. Son las mujeres quienes, generalmente, llevan la carga más importante en la dedicación de horas a cuidados, de tal manera que las instituciones deben poner especial atención al proponer un vínculo funcional con las familias, a fin de no exacerbar las inequidades que existen hoy en día. ${ }^{19}$ En la mayoría de los casos observados, las familias que atienden a un enfermo crónico o a un adulto mayor en el hogar se enfrentan a retos muy grandes, como por ejemplo la dificultad técnica de la atención en casa que implican muchas enfermedades. A este respecto, corresponde a las instituciones de salud revisar sus patrones de descarga de pacientes, así como establecer y consolidar los vínculos con las familias que permitan a estas últimas recibir el apoyo que solicitan. El fenómeno analizado en este trabajo también tendrá implicaciones sobre la forma de entender los sistemas de salud. Las propuestas teóricas actuales se enfocan a la descripción del sistema institucional y trabajan en un nivel general la descripción de la respuesta social organizada en el hogar y la comunidad, pero no se ocupan en describir la interfase existente entre el subsistema institucional y el resto del sistema, incluyendo la oferta de servicios no institucionales..$^{20}$ Es importante, por tanto, revisar estos preceptos, ya que la realidad cotidiana de la operación del sistema ha abierto enormes áreas a partir de la evolución de su respuesta ante nuevas necesidades que ameritan ser estudiadas y entendidas en su estructura y funcionamiento.

\section{Referencias}

I. Nigenda G, Matarazzo C, López-Ortega M. Los cuidados a la salud en el hogar: tendencias e inequidades. Análisis desde una perspectiva de género. México DF: FUNSALUD-SSA, 2005.

2. La Parra D. Contribución de las mujeres y los hogares más pobres a la producción de cuidados de salud informales. Gacet Sanit 200 I; 15:23-40. 3. Comas-Herrera A,Wittenberg R. European study of long-term care expenditure: Investigating the sensitivity of projections of future longterm care expenditure in Germany, Spain, Italy and the United Kingdom to changes in assumptions about demography, dependency, informal care, formal care and unit costs. Report to the European Commission, Employment and Social Affairs DG, PSSRU, Discussion Paper 1840. London: London School of Economics, 2003.

4. Comisión Económica para América Latina. Salud y envejecimiento. En: Las personas mayores en América Latina y el Caribe: diagnóstico sobre la situación y las políticas. Santiago de Chile: CEPAL, 2003.

5. Esteban ML. Cuidado y salud: costes para la salud de las mujeres y beneficios sociales. Género y cuidados: algunas ideas para la visibilización, el reconocimiento y la redistribución. En: SARE 2003, Cuidar cuesta: costes y beneficios del cuidado. Estado Español: Emakunde/Instituto Vasco de la Mujer. Euskadi, España, 2003.

6. García B, Blanco M, Pacheco E. Género y trabajo extradoméstico. En: García B, coord. Mujer, género y población en México. México DF: COLMEX, 1999.

7. Ariza M, De Oliveira O. Universo familiar y procesos demográficos. En: De Oliveira O,Ariza M, coords. Imágenes de la familia en el cambio de siglo. México, DF: UNAM, 2004.

8. Consejo Nacional de Población. Situación demográfica de México. México DF: CONAPO, 2003.

9. Instituto Nacional de Estadística, Geografía e Informática. Encuesta Nacional Sobre Uso del Tiempo (ENUT). México DF: INEGI, 2002. 10. Frenk J. Reformar sin deformar: la necesidad de una visión integral en la transformación del sistema de salud mexicano. En: Frenk J, ed. Observatorio de la salud. México, DF: FUNSALUD, 1997.

II. Pedrero-Nieto M. El trabajo doméstico no remunerado en México. Una estimación de su valor económico a través de la Encuesta Nacional sobre Uso del Tiempo 2002. México DF: Instituto Nacional de las Mujeres, 2005

12. Secretaría de Salud. Programa de Acción:Atención al Envejecimiento. México DF: SSA, 200I.

13. Instituto de Seguridad y Servicios Sociales para los Trabajadores del Estado. Escuela Nacional sobre Estudios y Atención al Envejecimiento. Programas de Estudio. México DF: ISSSTE, 2003.

14. Encuesta Nacional sobre Uso del Tiempo (ENUT). México DF: INEGI, 2002.

I5. Encuesta Nacional de Ingreso y Gasto de los Hogares. México DF: INEGI, 2002.

16. Organización de las Naciones Unidas. Panorama social de América Latina 2005. Santiago de Chile: ONU, 2006.

17. Organización para la Cooperación y el Desarrollo Económico. Estudios de la OCDE sobre los Sistemas de Salud. México DF: OCDE, 2005.

18. Knaul F, Nigenda G, Ramírez MA, Torres AC, Aguilar AM, LópezOrtega $M$ et al. Long-term care. Case study: Mexico. En: Brodsky J, Habib J, Hirshfeld M, eds. Long-term care in developing countries: ten case-studies. Ginebra:WHO, 2003.

19. García B, Blanco M, Pacheco E. Género y trabajo extradoméstico. En: García B, coord. Mujer, género y población en México. México DF: COLMEX, 1999.

20.WHO.The World Health Report 2000. Health Systems: Improving performance. Ginebra:WHO, 2000. 